\title{
Bladder Diverticula Caused by Occipital Horn Syndrome: A Case Report
}

\author{
Iva Palčić ${ }^{1}$, Andrea Cvitković Roić ${ }^{1,2}$, Alemka Jaklin Kekez ${ }^{1}$, Goran Roić ${ }^{3}$
}

${ }^{1}$ Helena Clinic for Pediatric Diseases

Zagreb, Croatia, ${ }^{2}$ Josip Juraj Strossmayer University of Osijek, Faculty of Medicine

${ }^{3}$ Children's Hospital Zagreb, Zagreb

Croatia

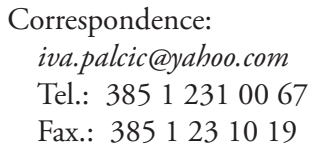

Received: January 15, 2019

Accepted: February 2, 2019

Key Words: Bladder Diverticula - Occipital Horn Syndrome - Children.

\begin{abstract}
Objective - To present a case of a bladder diverticula that was found to be a part of occipital horn syndrome, a rare X-linked recessive connective tissue disorder due to a copper transport defect (OMIM 304150). The syndrome is considered to be a milder variant of Menkes disease, with variable prognosis but substantially longer life expectancy. Case reports - We report the case of an eight-year-old boy with occipital horn syndrome who has a large bladder diverticula as a part of a connective tissue disorder. Diagnosis was based on the clinical features (hypotonia, hypotrophia, hyperextensible joints, hyperelastic, bruisable skin, psychomotoric development delay, recurrent UTI, large bladder diverticula), supported by low serum copper and ceruloplasmin levels, and confirmed by identification of mutations in the ATP7A gene (duplication in exons 11 and 12) at the age of 2 years. Conclusion - Large bladder diverticula can be a part of an underlying connective tissue disorder. Our case showed a boy with large bladder diverticula as part of occipital horn syndrome, a rare X-linked connective tissue disorder caused by a deficiency in the transport of copper, associated with mutations in the ATP7A gene. As bladder diverticula can be part of an underlying connective tissue disorder, it should be excluded in such patients presenting with a urinary tract infection.
\end{abstract}

\section{Introduction}

Occipital horn syndrome (OHS) is a rare $\mathrm{X}$-linked recessive disorder caused by mutations of the ATP7A gene (OMIM 304150) encoding a copper-transport protein, $\mathrm{Cu} 2+-$ transporting ATPase-alpha polypeptide. It is characterized by connective tissue disorders due to a copper transport defect. The syndrome is considered a milder variant of Menkes disease, with variable prognosis but substantially longer life expectancy. Since the disorder is X-linked recessive, the disease affects more males $(1,2)$.

Onset may occur from birth to early adulthood. Pregnancy is usually normal, but at birth children may present with hypotonia, hypothermia, jaundice or/and feeding problems. The first remarkable signs may be intractable diarrhoea, bladder diverticula or recurrent urinary tract infections. Motor development is delayed due to muscle hypotonia. The joints are hyperextensible and the skin is hyperelastic and bruisable. Unusual facial features include a long, thin face with a high forehead, down-slanting eyes, a hooked or prominent nose, a long philtrum, a high arched palate, and large prominent ears. The hair is usually not abnormal, although some patients may have dull and unusually coarse hair. Inguinal hernia and its recurrence are 
common. Vascular anomalies, such as varicose veins and arterial aneurysms have also been described. Intellectual capacity is low to borderline normal. Deformations in the skeleton, such as thoracolumbar kyphosis or scoliosis, and pectus deformity are usually present. Radiography shows characteristic occipital horns which are symmetric exostoses, protruding from the occipital bone, hence the name. These exostoses are usually detected around 5-10 years of age (3).

Diagnosis is based on the clinical features and confirmed by identification of a mutation in the ATP7A gene (4). Early parenteral copper-histidine supplementation may modify the disease's progression. However, copper histidine injections have been shown to be ineffective for treating the connective tissue manifestations of $\mathrm{OHS}(5,6)$.

Here we report a case of bladder diverticula that was found to be part of occipital horn syndrome.

\section{Case report}

A boy with macrohematuria and recurrent UTI was referred to our institution for the first time at the age of 14 months. He was born to a healthy non-consanguineous couple by spontaneous vaginal delivery $(37+$ 5 weeks of gestation). His birth weight was $2880 \mathrm{~g}$, birth length was $49 \mathrm{~cm}$, and head circumference was $36 \mathrm{~cm}$. The Apgar score was normal. There was no relevant family history, and three regular prenatal ultrasound check-ups were normal.

He presented at birth with poor muscle tone and a fracture of the occipital bone (Fig. 1) Upon admission the boy was hypotrophic and hypotonic with signs of delayed psychomotor development. He had a long thin face, a high forehead, down-slanting eyes and a high arched palate. His skin was hyperelastic and his joints hyperextensible. Urine analysis showed macrohaematuria with leukocyturia and bacteriuria on microscopic examination. Renal function was normal and macrohematuria disappeared in 2 days after introduction of antibiotic therapy. We concluded that this was a part of the E coli UTI isolated in the urinary culture. Ultrasound (US) examination revealed normal kidneys and a massive bladder diverticulum on the left contour, which was confirmed by voiding cystourethrogram (VCUG) (Fig. 2a, 2b, 2c). There was no evidence of bladder outlet obstruction or vesicoureteral reflux on VCUG. The urodynamics were normal. Due to his hypotonia and dysmorphic features, genetic and neuropaediatric counselling were performed. In laboratory findings low copper and ceruloplasmin were found. Genetic analysis showed mutation of the ATP7A gene (duplication in exons 11 and 12) and a diagnosis of occipital horn syndrome was made.

During the first year of follow-up the diverticulum did not empty properly and the boy suffered numerous UTIs, despite prophylactic antibiotics. At the age of 3 years we started clean intermittent catheterisation, and this was helpful for 2 years. However, at the age of 5 years the urinary infections started occurring once again and a new diverticu-

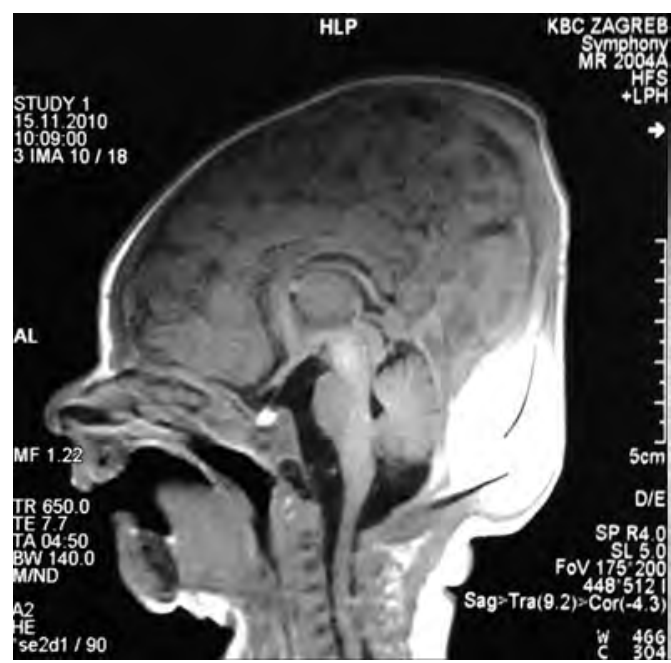

Fig 1. MRI of the Skull: Occipital Bone Fracture with Epidural and Subdural Haematoma. 

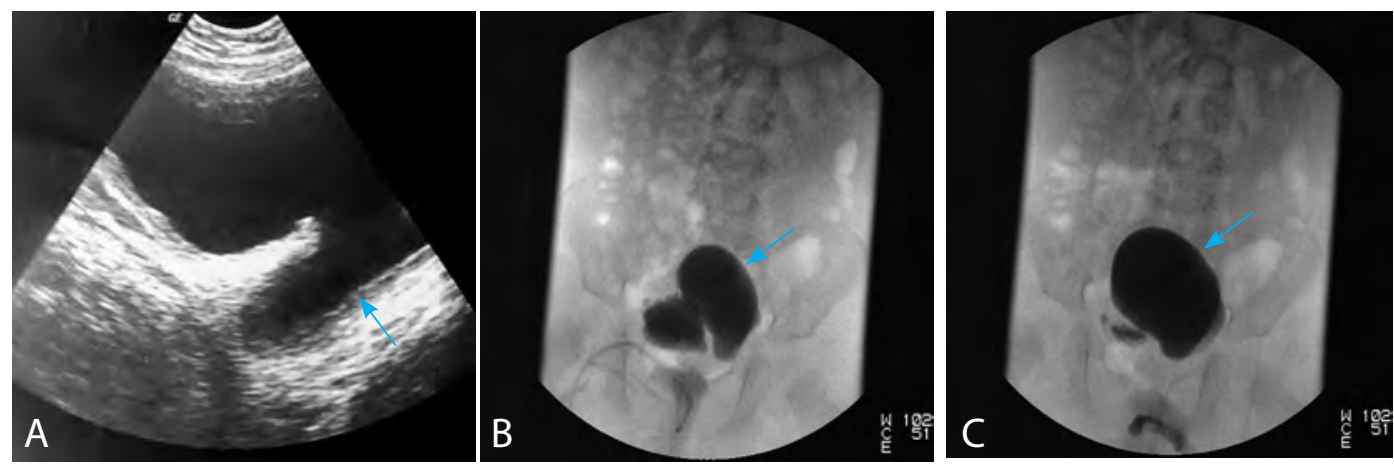

Fig. 2. Age $14^{\text {th }}$ Months. A. Bladder Ultrasound: Large Diverticulum of the Left Contour; Voiding Cystourethrogram: Large Diverticulum of the Left Contour (B) and its Poor Emptying During Voiding (C).

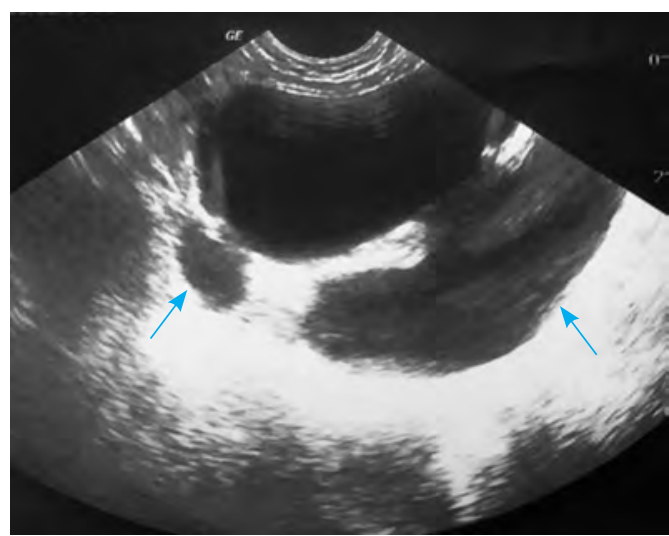

Fig. 3. Age 5 Years. Bladder Ultrasound: Large Diverticulum of the Left Contour and a New One on the Right Side.

lum, now on the right side, appeared (Fig. 3). As a result the boy underwent cystostomy at the age of 6 years. The boy is now 8 years old and he has been free of UTI for two years.

\section{Discussion}

Bladder diverticula are defined as the herniation of the bladder mucosa through muscular fibres of the bladder wall, resulting in a thinwalled structure that empties poorly during micturition, hence its most common presentation is a UTI. The causes are numerous, and diverticula may be classified into primary, congenital diverticula and secondary diverticula due to bladder outlet obstruction, or diverticula secondary to connective tissue or muscle fragility (7). The latter is seen in disease entities such as Prune belly syndrome, Ehlers-Danlos syndrome, OHS, Menkes disease and Williams-Beuren syndrome.

We report a case of bladder diverticula that was found to be a part of occipital horn syndrome $(1,8)$. Complete loss of function caused by mutations in ATP7A lead to Menkes disease, involving impaired brain development, neurological degeneration, connective tissue abnormalities, and high mortality in early infancy (9). Less severe defects produce the allelic disorder occipital horn syndrome (10). The latter phenotype involves mainly connective tissue problems, including skin and joint laxity, tortuous blood vessels, bladder diverticula and hernias, and also skeletal abnormalities that include occipital exostoses, which give rise to the syndrome's name $(2,3)$.

One Korean study showed that urological problems occurred frequently in Menkes disease, with bladder diverticula being the most common (11). Bladder diverticula result in urinary stasis with a high residual urine volume, which leads to UTIs. Therefore, the main goal of management in patients with bladder diverticula is to accomplish complete bladder emptying with clean intermittent catheterization, or open surgical drainage in severe cases. 
In our patient clean intermittent catheterisation (CIC) and prophylactic antibiotics were helpful to prevent UTIs for two years. At the age of 5 years a new diverticulum appeared and recurrent infections could not be controlled despite CIC and prophylactic antibiotics, therefore it was necessary to place a cystostoma at the age of 6 years. Finally, for the past two years he has been free of UTI. Our patient also had am occipital bone fracture at birth which we found. This was due to a traumatic birth event because bone fragility is a not part of occipital horn syndrome (12).

\section{Conclusion}

Our case showed a boy with large bladder diverticula in occipital horn syndrome, a rare $\mathrm{X}$-linked connective tissue disorder caused by a deficiency in the transport of copper, associated with mutations in the ATP7A gene (OMIM 304150). As bladder diverticula may be a part of underlying connective tissue disorder, it should be excluded in such patients presenting with urinary tract infections. A multidisciplinary approach is important for early and correct diagnosis in order to start appropriate treatment and follow up.

Conflict of Interest: The authors declare that they have no conflict of interest.

\section{Reference}

1. Yasmeen S, Lund K, De Paepe A, De Bie S, Heiberg A, Silva J, et al. Occipital horn syndrome and classical Menkes Syndrome caused by deep intronic mutations, leading to the activation of ATP7A pseudo-exon. Eur J Hum Genet. 2014;22(4):51721.

2. Tang J, Robertson S, Lem KE, Godwin SC, Kaler SG. Functional copper transport explains neuro- logic sparing in occipital horn syndrome. Genet Med. 2006;8(11):711-8.

3. Bazzocchi A, Femia R, Feraco P, Battista G, Canini R, Guglielmi G. Occipital horn syndrome in a woman: skeletal radiological findings. Skeletal Radiol. 2011;40(11):1491-4.

4. Das S, Levinson B, Vulpe C, Whitney S, Gitschier J, Packman S. Similar splicing mutations of the Menkes/mottled copper-transporting ATPase gene in occipital horn syndrome and the blotchy mouse. Am J Hum Genet. 1995;56:570-6.

5. Yoganathan S, Sudhakar SV, Arunachal G, Thomas M, Subramanian A, George R. et al. Menkes disease and response to copper histidine: An Indian case series. Ann Indian Acad Neurol. 2017;20(1):62-8.

6. Kodama H, Fujisawa C, Bhadhprasit W. Pathology, clinical features and treatments of congenital copper metabolic disorders--focus on neurologic aspects. Brain Dev. 2011;33(3):243-51.

7. Legros L, Revencu N, Nassogne MC, Wese FX, Feyaerts A. Multiple bladder diverticula caused by occipital horn syndrome. Arch Pediatr. 2015;22(11):1147-50.

8. Horn N, Tümer Z. Menkes disease and the occipital horn syndrome. In: Royce P M, Steinman B, editors. Connective Tissue and Its Heritable Disorders. Wiley-Liss, Inc.; New York: 2002. pp. 651685.

9. Vairo FPE, Chwal BC, Perini S, Ferreira MAP, de Freitas Lopes AC, Saute JA. A systematic review and evidence-based guideline for diagnosis and treatment of Menkes disease. Mol Genet Metab. 2019;126(1):6-13.

10. Kaler SG, Gallo LK, Proud VK, Percy AK, Mark Y, Segal NA, et al. Occipital horn syndrome and a mild Menkes phenotype associated with splice site mutations at the MNK locus. Nat Genet. 1994;8(2):195-202.

11. Kim MY, Kim JH, Cho MH, Choi YH, Kim SH, Im YJ et al. Urological Problems in Patients with Menkes Disease. J Korean Med Sci. 2018;34(1):e4.

12. Arifin M.Z, Gill A.S, Anwar A.D, Djuwantono T, Faried A. Spontaneous depressed skull fracture during vaginal delivery: A report of two cases and literature review. Ind. J. Neurotrauma. 2013;10:33-7. 